\title{
Acetazolamide, Nifedipine and Phosphodiesterase Inhibitors: Rationale for Their Utilization as Adjunctive Countermeasures in the Treatment of Coronavirus Disease 2019 (COVID-19)
}

\author{
Isaac Solaimanzadeh ${ }^{1}$ \\ 1. Internal Medicine, Interfaith Medical Center, Brooklyn, USA \\ Corresponding author: Isaac Solaimanzadeh, isolaimanzadeh@interfaithmedical.org
}

\begin{abstract}
Effective treatments for Coronavirus Disease 2019 (COVID-19) outbreak are urgently needed. While antiviral approaches and vaccines are being considered immediate countermeasures are unavailable. The aim of this article is to outline a perspective on the pathophysiology of COVID-19 in the context of the currently available clinical data published in the literature. This article appreciates clinical data published on COVID19 in the context of another respiratory illness - high altitude pulmonary edema (HAPE). Both conditions have significant similarities that portend pathophysiologic trajectories. Following this potential treatment options emerge.
\end{abstract}

Both COVID-19 and HAPE exhibit a decreased ratio of arterial oxygen partial pressure to fractional inspired oxygen with concomitant hypoxia and tachypnea. There also appears to be a tendency for low carbon dioxide levels in both as well. Radiologic findings of ground glass opacities are present in up to $86 \%$ of patients with COVID-19 in addition to patchy infiltrates. Patients with HAPE also exhibit patchy infiltrates throughout the pulmonary fields, often in an asymmetric pattern and CT findings reveal increased lung markings and ground glass-like changes as well. Widespread ground-glass opacities are most commonly a manifestation of hydrostatic pulmonary edema. Similarly, elevated fibrinogen levels in both conditions are likely an epiphenomenon of edema formation rather than coagulation activation. Autopsy results of a COVID-19 fatality revealed bilateral diffuse alveolar damage associated with pulmonary edema, pro-inflammatory concentrates, and indications of early-phase acute respiratory distress syndrome (ARDS). HAPE itself is initially caused by an increase in pulmonary capillary pressure and induces altered alveolar-capillary permeability via high pulmonary artery hydrostatic pressures that lead to a protein-rich and mildly hemorrhagic edema. It appears that COVID-19 and HAPE both discretely converge on ARDS. In light of this, a countermeasure that has been shown to be effective in the analogous condition of HAPE is Acetazolamide. Acetazolamide has a myriad of effects on different organ systems, potently reduces hypoxic pulmonary vasoconstriction, improves minute ventilation and expired vital capacity. Other therapeutics to consider that are also directed towards decreased pulmonary pressure include Nifedipine and Phosphodiesterase inhibitors.

Received 03/07/2020

Review began 03/09/2020 Review ended 03/16/2020 Published 03/20/2020

๑) Copyright 2020

Solaimanzadeh. This is an open access article distributed under the terms of the Creative Commons Attribution License CC-BY 4.0., which permits unrestricted use, distribution, and reproduction in any medium, provided the original author and source are credited.
This review describes COVID-19 in parallel to HAPE. Deranged respiratory parameters that are present in both conditions are highlighted. The utilization of medications found to be effective in HAPE, for the treatment of COVID-19, is proposed. Given the medical emergency of a growing contagion and the thousands of lives at stake, expedient attempts to improve survival are needed. Acetazolamide, Nifedipine and Phosphodiesterase inhibitors may be potential countermeasures.

Categories: Infectious Disease, Pulmonology, Public Health

Keywords: coronavirus, covid-19, high altitude pulmonary edema, respiratory care, wuhan coronavirus, acetazolamide, ground glass opacities, hypoxia, covid-2019, novel coronavirus

\section{Introduction And Background}

Effective treatments for Coronavirus Disease 2019 (COVID-19) outbreak are urgently needed. While antiviral approaches are being considered and trials as well as vaccines may be forthcoming, immediate countermeasures are still remiss [1].

In ideal circumstances medications are intentionally designed, profiled and tested to combat initiators of pathophysiologic processes. However, when that is not available, there may be a need to consider treatment regimens from analogous disease patterns. Matching clinical dispositions can be considered in efforts to develop therapeutic interventions. Moreover, dire outcomes of illness may be overcome with adjunctive measures that do not necessarily cure underlying disease. Rather, supportive care as well as adjunctive countermeasures may assist patients in surviving viral illness. 
currently available clinical data published in the literature. Following a characterization of the disease vis-avis a similar respiratory illness, potential treatment options may emerge.

\section{Review}

Supportive management with specific Respiratory and Ventilator support are current mainstays of treatment [2]. Sequential progression of respiratory compromise has been observed - highlighting the primacy of respiratory malfunction in overall clinical demise [3].

Therefore, garnering management approaches from similar respiratory conditions may be beneficial. Analyzing clinical data reported in published studies reveal striking similarities to high altitude pulmonary edema (HAPE) as manifested during the acute hypoxic ventilatory response.

To begin with, in severe cases, both COVID-19 and HAPE exhibit a decreased ratio of arterial oxygen partial pressure to fractional inspired oxygen (Pao2:FiO2 ratio) with concomitant hypoxia and tachypnea $[4,5]$. There also appears to be a tendency for low carbon dioxide levels in COVID-19 as the median partial pressure of carbon dioxide (PaCO2) level was $34 \mathrm{mmHg}$ (inter-quartile range: 30-38; normal range: 35-48) in a recent JAMA article describing 138 hospitalized cases [6]. Initial exposure to hypoxia at high altitude leads to an immediate increase in ventilation that blows off large quantities of carbon dioxide, producing hypocapnia as well [7]. Furthermore, blood gases of non-acclimatized mountaineers with severe illness were accompanied by a significant decrease in arterial oxygen due to an increase in alveolar-arterial oxygen difference, although herein arterial $\mathrm{PaCO} 2$ did not change significantly [8]. In short, hypoxia and hypocapnia are seen in both conditions, but there is more.

Radiologic findings of ground-glass opacities are present in up to $86 \%$ of patients with COVID-19 with $76 \%$ having bilateral distribution and 33\% peripheral [9]. Notably, lung cavitations, discrete pulmonary nodules, pleural effusions, and lymphadenopathy were absent [10]. In addition to this, patchy infiltrates are present [11]. Patients with HAPE also exhibit patchy infiltrates throughout the pulmonary fields, often in an asymmetric pattern and CT findings reveal increased lung markings and ground glass-like changes as well [12-14]. It has been shown that widespread ground-glass opacities are most commonly a manifestation of hydrostatic pulmonary edema and this is a central point to consider going forward [15].

See, all older patients in a familial cluster had elevated fibrinogen levels [16]. In tandem, markers of fibrin formation were significantly elevated in HAPE and Fibrin generation in that condition is deliberated as an epiphenomenon of edema formation rather than coagulation activation [8]. Altogether, these specific pulmonary clinical manifestations exhibit identical features between both COVID-19 and HAPE.

There certainly is much to ascertain with regard to the precise pathophysiology of COVID-19. Investigation of virulent properties of COVID-19 as well inflammatory responses and their effects on Alveolar integrity requires further study. Autopsy results of a COVID-19 fatality revealed bilateral diffuse alveolar damage associated with pulmonary edema, pro-inflammatory concentrates, and indications of early-phase acute respiratory distress syndrome (ARDS) [17]. HAPE itself is initially caused by an increase in pulmonary capillary pressure [18]. HAPE induces altered alveolar-capillary permeability via high pulmonary artery hydrostatic pressures that lead to a protein-rich and mildly hemorrhagic edema [19]. COVID-19 and HAPE both discretely converge on ARDS [5,17].

Yet, it can be posited that beginning early treatment may prevent ARDS development. Regardless of pathophysiologic triggers, stark clinical endpoints are apparent and similar in nature. Ultimately, distinctive pulmonary specific parameters in severe disease have comparable patterns (Table 1). 


\section{Cureus}

\begin{tabular}{|l|l|l|}
\hline Parameter & HAPE & COVID-19 \\
\hline Pao2:FiO2 ratio & Decreased & Decreased \\
\hline Hypoxia & Present & Present \\
\hline Tachypnea & Increased & Increased \\
PaCO2 level & Decreased & Decreased \\
\hline Ground Glass Opacities on Chest CT & Present & Present \\
\hline Patchy Infiltrates on Chest X-RAY & Present & Present \\
\hline Fibrinogen levels/Fibrin formation & Increased & Increased \\
Alveolar compromise & Present & Present \\
Acute Respiratory Distress Syndrome Development in Severe Disease & Present & Present \\
\hline
\end{tabular}

\section{TABLE 1: Similar patterns of pulmonary disease between HAPE and COVID-19}

HAPE: High altitude pulmonary edema; COVID-19: Coronavirus disease 2019; Pao2:FiO2 ratio: Arterial oxygen partial pressure to fractional inspired oxygen ratio; PaCO2 level: Partial pressure of carbon dioxide; Chest CT: Computed tomography of chest.

In light of this, a countermeasure that has been shown to be effective in high altitude illness is Acetazolamide.

Acetazolamide has a myriad of effects on different organ systems [20]. It potently reduces hypoxic pulmonary vasoconstriction [21]. Improved minute ventilation and expired vital capacity has been shown in climbers taking Acetazolamide as well [22].

Furthermore, over $70 \%$ of patients with COVID-19 had elevated lactate dehydrogenase levels [23]; this too may be connected to hypoxia. Evidently, Acetazolamide has physiologic effects that delay plasma lactate appearance with no effect on ventilatory threshold [24].

Other therapeutics that have been shown to be effective in the analogous condition of HAPE and that are directed towards decreased pulmonary pressure include Nifedipine and Phosphodiesterase inhibitors (Table 2) $[25,26]$.

\section{Acetazolamide}

Nifedipine

Sildenafil

Tadalafil

\section{$250 \mathrm{mg}$ every 12 hours}

$30 \mathrm{mg}$ extended release every 12 hours

20-50 mg every 8 hours

$10 \mathrm{mg}$ every 12 hours

\section{TABLE 2: Sample medications and dosages utilized in high altitude illness and HAPE}

\section{Conclusions}

This review describes COVID-19 in parallel to HAPE. Deranged respiratory parameters that are present in both conditions are highlighted. The utilization of medications found to be effective in HAPE for the treatment of COVID-19 is proposed. Given the medical emergency of a growing contagion and the thousands of lives at stake, expedient attempts to improve survival are needed. Acetazolamide, Nifedipine and Phosphodiesterase inhibitors may present an opportunity for countermeasure development.

\section{Additional Information}

Disclosures 
Conflicts of interest: In compliance with the ICMJE uniform disclosure form, all authors declare the following: Payment/services info: All authors have declared that no financial support was received from any organization for the submitted work. Financial relationships: All authors have declared that they have no financial relationships at present or within the previous three years with any organizations that might have an interest in the submitted work. Other relationships: All authors have declared that there are no other relationships or activities that could appear to have influenced the submitted work.

\section{References}

1. Li G, De Clercq E: Therapeutic options for the 2019 novel coronavirus (2019-nCoV). Nat Rev Drug Discov. 2020, 19:149-150. 10.1038/d41573-020-00016-0

2. Arabi YM, Fowler R, Hayden FG: Critical care management of adults with community-acquired severe respiratory viral infection. Intensive Care Med. 2020, 46:315-328. 10.1007/s00134-020-05943-5

3. Huang C, Wang Y, Li X, et al.: Clinical features of patients infected with 2019 novel coronavirus in Wuhan, China. Lancet. 2020, 395:497-506. 10.1016/S0140-6736(20)30183-5

4. Wang M, Zhou Y, Zong Z, et al.: A precision medicine approach to managing 2019 novel coronavirus pneumonia. Precis Clin Med. 2020, pbaa002:10.1093/pcmedi/pbaa002

5. Ma SQ, Wu TY, Cheng Q, Li P, Bian H: Acute respiratory distress syndrome secondary to high-altitude pulmonary edema: a diagnostic study. J Med Lab Diagn. 2013, 4:1-7. 10.5897/JMLD12.007

6. Wang D, Hu B, Hu C, et al.: Clinical characteristics of 138 hospitalized patients with 2019 novel coronavirus-infected pneumonia in Wuhan, China. JAMA. 2020, 323:1061-1069. 10.1001/jama.2020.1585

7. Taylor AT: High-altitude illnesses: physiology, risk factors, prevention and treatment. Rambam Maimonides Med J. 2011, 2:e0022. 10.5041/RMMJ.10022

8. Bartsch PE, Waber U, Haeberli AN, Maggiorini M, Kriemler S, Oelz O, Straub WP: Enhanced fibrin formation in high-altitude pulmonary edema. J Appl Physiol. 1987, 63:752-757. 10.1152/jappl.1987.63.2.752

9. Kanne JP: Chest CT findings in 2019 novel coronavirus (2019-nCoV) infections from Wuhan, China: key points for the radiologist. Radiology. 2020, 295:200241. 10.1148/radiol.2020200241

10. Chung M, Bernheim A, Mei X, et al.: CT imaging features of 2019 novel coronavirus (2019-nCoV) . Radiology. 2020, 295:200230. 10.1148/radiol.2020200230

11. Pan Y, Guan H, Zhou S, et al.: Initial CT findings and temporal changes in patients with the novel coronavirus pneumonia (2019-nCoV): a study of 63 patients in Wuhan, China. (Epub ahead of print). Eur Radiol. 2020, 10.1007/s00330-020-06731-x

12. Kobayashi T, Koyama S, Kubo K, Fukushima M, Kusama S: Clinical features of patients with high-altitude pulmonary edema in Japan. Chest. 1987, 92:814-821. 10.1378/chest.92.5.814

13. Bärtsch P, Swenson ER, Maggiorini M: Update: high altitude pulmonary edema. Adv Exp Med Biol. 2001, 502:89-106. 10.1007/978-1-4757-3401-0_8

14. Zhou Q: Standardization of methods for early diagnosis and on-site treatment of high-altitude pulmonary edema. Pulm Med. 2011, 2011:7. 10.1155/2011/190648

15. Hewitt MG, Miller WT Jr, Reilly TJ, Simpson S: The relative frequencies of causes of widespread groundglass opacity: a retrospective cohort. Eur J Radiol. 2014, 83:1970-1976. 10.1016/j.ejrad.2014.06.025

16. Chan JF, Yuan S, Kok KH, et al.: A familial cluster of pneumonia associated with the 2019 novel coronavirus indicating person-to-person transmission: a study of a family cluster. Lancet. 2020, 395:514-523. 10.1016/S0140-6736(20)30154-9

17. Xu Z, Shi L, Wang Y, et al.: Pathological findings of COVID-19 associated with acute respiratory distress syndrome. (Epub ahead of print). Lancet Respir Med. 2020, 10.1016/S2213-2600(20)30076-X

18. Maggiorini M, Mélot C, Pierre S, et al.: High-altitude pulmonary edema is initially caused by an increase in capillary pressure. Circulation. 2001, 103:2078-2083. 10.1161/01.CIR.103.16.2078

19. Swenson ER, Maggiorini M, Mongovin S, Gibbs JSR, Greve I, Mairbäurl H, Bärtsch P: Pathogenesis of highaltitude pulmonary edema: inflammation is not an etiologic factor. JAMA. 2002, 287:2228-2235. 10.1001/jama.287.17.2228

20. Leaf DE, Goldfarb DS: Mechanisms of action of acetazolamide in the prophylaxis and treatment of acute mountain sickness. J Appl Physiol. 2007, 102:1313-1322. 10.1152/japplphysiol.01572.2005

21. Swenson ER: Carbonic anhydrase inhibitors and hypoxic pulmonary vasoconstriction. Respir Physiol Neurobiol. 2006, 151:209-216. 10.1016/j.resp.2005.10.011

22. Larson EB, Roach RC, Schoene RB, Hornbein TF: Acute mountain sickness and acetazolamide: clinical efficacy and effect on ventilation. JAMA. 1982, 248:328-332. 10.1001/jama.1982.03330030034021

23. Chen N, Zhou M, Dong X, et al.: Epidemiological and clinical characteristics of 99 cases of 2019 novel coronavirus pneumonia in Wuhan, China: a descriptive study. Lancet. 2020, 395:507-513. 10.1016/S01406736(20)30211-7

24. Scheuermann BW, Kowalchuk JM, Paterson DH, Cunningham DA: Carbonic anhydrase inhibition delays plasma lactate appearance with no effect on ventilatory threshold. J Appl Physiol. 2000, 88:713-721. 10.1152/jappl.2000.88.2.713

25. Bartsch P, Maggiorini M, Ritter M, Noti C, Vock P, Oelz O: Prevention of high-altitude pulmonary edema by nifedipine. N Engl J Med. 1991, 325:1284-1289. 10.1056/NEJM199110313251805

26. Maggiorini M: Prevention and treatment of high-altitude pulmonary edema. Prog Cardiovasc Dis. 2010, 52:500-506. 10.1016/j.pcad.2010.03.001 